Meta

Journal des traducteurs

Translators' Journal

\title{
Quelques certitudes sur la préservation de l'incertitude dans le texte traduit
}

\section{Muguraş Constantinescu}

Volume 61, numéro 1, mai 2016

Des zones d'incertitudes en traduction

URI : https://id.erudit.org/iderudit/1036989ar

DOI : https://doi.org/10.7202/1036989ar

Aller au sommaire du numéro

\section{Éditeur(s)}

Les Presses de l’Université de Montréal

ISSN

0026-0452 (imprimé)

1492-1421 (numérique)

Découvrir la revue

Citer cet article

Constantinescu, M. (2016). Quelques certitudes sur la préservation de l'incertitude dans le texte traduit. Meta, 61(1), 187-203.

https://doi.org/10.7202/1036989ar
Résumé de l'article

Nous proposons dans notre article une réflexion sur les stratégies de préservation de l'ambiguïté - globale, ponctuelle ou totale - dans un texte littéraire, notamment dans un texte poétique traduit, accueilli par une autre culture. Comme on le sait, le texte poétique en particulier et le texte littéraire en général exploitent parfois l'ambiguïté, l'obscurité et la plurivalence des sens ; le défi pour le traducteur sera alors de les garder, leur préservation étant garante de toutes les lectures possibles. En partant de plusieurs traductions de poèmes de Mallarmé, Valéry et Tzara du français vers le roumain et des auto-traductions du roumain vers le français de Luca, nous identifierons les difficultés récurrentes que le traducteur doit vaincre et nous analyserons les divers procédés et stratégies utilisés dans ce but. Face aux pièges de l'ambiguïté, il reste au traducteur comme attitude et stratégie adéquates l'exploration à fond des potentialités de la langue traduisante, conduisant à l'éveil des termes rares ou anciens ou même à la trouvaille ; à cela s'ajoute une créativité au second degré, bien maîtrisée par les contraintes de l'original. 


\title{
Quelques certitudes sur la préservation de l'incertitude dans le texte traduit*
}

\author{
MUGURAŞ CONSTANTINESCU \\ Universitatea Ștefan cel Mare, Suceava, Roumanie \\ mugurasc@gmail.com
}

\begin{abstract}
RÉSUMÉ
Nous proposons dans notre article une réflexion sur les stratégies de préservation de l'ambiguïté - globale, ponctuelle ou totale - dans un texte littéraire, notamment dans un texte poétique traduit, accueilli par une autre culture. Comme on le sait, le texte poétique en particulier et le texte littéraire en général exploitent parfois l'ambiguïté, l'obscurité et la plurivalence des sens; le défi pour le traducteur sera alors de les garder, leur préservation étant garante de toutes les lectures possibles. En partant de plusieurs traductions de poèmes de Mallarmé, Valéry et Tzara du français vers le roumain et des auto-traductions du roumain vers le français de Luca, nous identifierons les difficultés récurrentes que le traducteur doit vaincre et nous analyserons les divers procédés et stratégies utilisés dans ce but. Face aux pièges de l'ambiguïté, il reste au traducteur comme attitude et stratégie adéquates l'exploration à fond des potentialités de la langue traduisante, conduisant à l'éveil des termes rares ou anciens ou même à la trouvaille; à cela s'ajoute une créativité au second degré, bien maîtrisée par les contraintes de l'original.
\end{abstract}

\section{ABSTRACT}

The aim of the present paper is to reflect upon the strategies of preserving global as well as specific or total ambiguity in a literary text (a poetic text in particular) when translated and therefore imported by another culture. It is a well-known fact that literary texts in general, and poetic texts in particular, sometimes make use of ambiguity, obscurity and plurivalence of meaning, which represent a real challenge for the translator, who is supposed to preserve them and ensure all potentialities. Starting from a series of Romanian versions of poems by Mallarmé, Valéry and Tzara as well as some of Luca's self-translations (from Romanian into French), we will identify the recurring difficulties the translator is faced with and the solutions he finds. While facing the traps of ambiguity, the translator is left to explore the potential of the target language, which sometimes implies resorting to rare, archaic terms or even "trouvailles" and a second-degree creativity restricted by what(ever) is imposed by the original.

\section{MOTS-CLÉS/KEYWORDS}

texte poétique, ambiguïté, obscurité, plurivalence des sens, lectures possibles poetic text, ambiguity, obscurity, multivalence of meaning, multiple readings

La démarche herméneutique est la stratégie la plus inappropriée, je pense, quand on est confronté, en tant que traducteurs, à un texte de poéticité maximale, autrement dit d'une ambiguïté maximale.

(Irina Mavrodin 2006a/2013: 102)

En 2005, l'École de traducteurs et d'interprètes de l'Université Saint-Joseph de Beyrouth avait lancé un débat autour de la nécessité de dissiper le flou en traduction et en traductologie qui semblaient connaître, à l'époque, une véritable «babélisation" 
(Abou Fadel et Awaiss 2005: 8). Des traductologues et traducteurs réputés comme Michel Ballard, Christian Balliu, Maria Carreras, André Clas, Elena de la Fuente, Jean Delisle, Hannelore Lee-Jahnke, Jarjoura Hardane, Hayssam Kotob, Jean-René Ladmiral, Charles Le Blanc, Marianne Lederer, Henri Meschonnic, Jean Peeters, Anthony Pym, Nadine Riachi, pour n'en énumérer que quelques-uns, ont répondu à l'appel formulé par Henri Awaiss et Gina Abou Fadel. Leurs réponses ont exprimé une diversité d'idées et de nuances: si Ladmiral a nettement dit «traduire c'est désambiguïser» (2005: 127), Clas a embrassé la métaphore pour soutenir que «traduire c'est tailler un diamant à multiples facettes» (2005: 49), tandis que Delisle a attiré l'attention sur une «complexité» théorique «à apprivoiser» (2005: 69) et Ballard sur le fait que la traduction d'un poème et celle d'une notice de montage ont peu de choses en commun (2005: 21). Les chercheurs et traducteurs qui ont donné des réponses, réunies dans le volume Pour dissiper le flou, ont souligné le type de traduction à prendre en compte, le danger d'une traductologie employée comme instrument de pouvoir (Balliu 2005: 27), celui d'une interprétation qui ne mène pas à la clarté (Le Blanc 2005: 131), celui du flou qui conduit à une traduction «effaçante» (Meschonnic 2005: 147). D’une façon ou d'une autre, les coauteurs de l'ouvrage ont donné raison aux initiateurs du débat qui, dans leur appel, reconnaissaient que le «flou» n'est pas toujours «néfaste ou négatif» et qu'«en science, en poésie, en art, il peut même être source de création» (Abou Fadel et Awaiss 2005: 9).

Ce dernier aspect nous préoccupe dans ce qui suit, surtout qu'il n'a pas été totalement absent dans la deuxième journée de «Traductologie de plein champ» organisée par l'Université Paris 7 sur les «zones d'incertitude en traduction». À cette occasion, plusieurs chercheurs ont fait l'éloge du "doute méthodique» dans la réflexion traductologique, en l'associant à l'incertitude, et des traductologues comme Esperanza Alarcón, Maribel Tercedor et Clara Inés López Rodríguez de l'Université de Grenade sont même arrivées à la conclusion que l'incertitude est « notre grande force cachée $»^{2}$.

Avec de telles prémisses, nous tenterons de démontrer que la préservation de l'incertitude, associée par nous à l'ambiguïté et à la plurivalence, est nécessaire et appropriée dans la traduction du texte littéraire, notamment poétique. Cela ne limite pas la difficulté de rendre les zones d'incertitudes - connotations, métaphores, nuances - au seul texte littéraire, car le texte non littéraire, pragmatique ou médical, en comporte lui aussi (Froeliger 2008: 77; Balliu 2010: 19), mais cette problématique dépasse le cadre de notre article.

\section{Considérations sur la préservation de l'incertitude et de l'ambiguïté du texte original}

Le mot «incertitude», choisi par les chercheurs de Paris, Bruxelles et Genève pour lancer le débat concernant sa place dans la traduction, est riche en synonymes, connotations et nuances qui le rendent difficile à cerner, car il renvoie, selon le contexte, pour ne pas dire la «zone» où il se trouve, en même temps ou, tour à tour, à hésitation, doute, flou, indécision, tâtonnement, obscurité, instabilité, confusion, fluctuation, oscillation. Il peut renvoyer aussi à des mots plus éloignés, à première vue, comme variabilité, souci, précarité, irrésolution, inconstance, flottement, mystère, énigme ou même vague, équivoque, et aller jusqu'à évoquer des vocables comme 
anxiété et vulnérabilité. Cette riche épaisseur de significations et suggestions montre, en fait, que ce mot, placé dans un entourage adéquat, notamment le syntagme «zones d'incertitude», est un bon et stimulant choix pour un débat traductologique « de plein champ».

D'ailleurs, dans l'histoire de la réflexion sur la traduction, on trouve des positions contre la production, à travers la traduction, d'un texte univoque pour rendre un texte littéraire qui, à l'origine, ne l'est pas. Walter Benjamin, par exemple, dans le prologue à sa version allemande des Tableaux parisiens, réfléchit à la «tâche du traducteur», censée assurer la «survie» des œuvres, tâche qu'il associe à un texte «énigmatique», «insaisissable» (Benjamin 1955/2000: 45).

Une telle idée appelle celle d'une (re)traduction qui garde presque la même richesse polysémique et à peu près les mêmes ambiguïtés que l'original et repousse la tentation d'expliciter et de clarifier le texte (re)traduit. Elle évoque aussi celle d'Umberto Eco, qui, dans son livre Dire presque la même chose, parle, tour à tour, du risque de produire de l'ambiguïté à partir d'un noud de tension du texte, de la difficulté, voire l'impossibilité, de maintenir l'ambiguïté dans le texte traduit, mais aussi de la volonté de la maintenir pour susciter une interprétation «oscillant entre deux alternatives» (Eco 2003/2008: 251).

On retrouve cette volonté d'incertitude et d'ambiguïté dans la "pratico-théorie» de la traduction qu'Irina Mavrodin (2006b: 170) élabore, par analogie à une lecture plurielle du texte original:

Je propose une théorie de la traduction en tant que «lecture plurielle» qui reste - paradoxalement - valide même lorsque, dans le cas des textes qui l'exigent, le traducteur essaie de maintenir l'ambiguité - la pluralité des sens donc - dans l'original. Choisir de maintenir l'ambiguïté est, en soi, l'option pour un certain type de lecture. [...] Le lecteur (tout comme le critique en tant que lecteur spécialisé) re-crée l'œuvre par sa lecture. Ce point de vue peut être élargi vers la traduction littéraire et vers l'activité du traducteur littéraire dans lequel on peut voir un lecteur dont l'effort, matérialisé dans la transposition de l'œuvre d'une langue à l'autre, est le plus proche de l'idée que nous nous faisons de la lecture en tant qu'acte créateur. (Mavrodin 2006a/2012d: 134) ${ }^{3}$

Professeure, traductrice et poète elle-même, Mavrodin appuie sa réflexion traductologique autant sur ses expériences de traduction et d'écriture que sur la connaissance de la poésie moderne, qu'elle considère comme la meilleure illustration d'un texte qui appelle une lecture/traduction plurielle:

La difficulté de maintenir l'ambiguïté (concept important de la poétique moderne) tout en la traduisant, [...] devient évidente quand on a affaire à des auteurs d'une ambiguïté maximale, comme Rimbaud, Mallarmé, Char, Michaux, etc. Le traducteur se trouve dans la situation d'inventer, de créer un texte isomorphe (autre concept de la poétique) au texte original, un texte qui fasse attention à sa constitution, à la manière dont il est constitué, qui lui permette une lecture plurivoque, et non pas univoque qu'il est tout le temps tenté de "chercher», de faire émerger par une démarche herméneutique. (Mavrodin 2006a/2013: 1 102) ${ }^{4}$

Les textes poétiques que nous prenons pour objet d'analyse ont cette marque d'une "poéticité maximale », qui s'associe ou s'identifie à une "ambiguïté maximale " (Mavrodin 2006a/2013: 102). Cette dernière est difficile à rendre à travers la traduction sans privilégier une certaine lecture du texte, surtout si la langue d'accueil n'a pas toujours les mêmes valeurs d'ambiguïté que celle de départ. 
Poète et traducteur à son tour, Ştefan Augustin Doinaş est préoccupé lui aussi par la poéticité et l'ambiguïté du texte à traduire, à « re-créer» dans une autre «langue poétique» (1974b : 258); comme il s'attaque à des textes de Mallarmé et de Valéry qui cherchent également la perfection formelle du texte, le traducteur roumain reproche au premier d'avoir traduit en prose des poésies de Poe et d'avoir ainsi sacrifié la forme proprement poétique de l'original. Il ne suit pas ce qu'on a appelé la «leçon de Mallarmé» (Bonnefoy 2000 : 51), car, selon Doinaş, enlever à un sonnet, en le traduisant, sa «forme» de sonnet signifie lui enlever une part de sa spécificité poétique, d'où l'attention qu'il accorde au «son» (1974b : 259-260). Mais le poète-traducteur ne limite pas son travail au seul signifiant et envisage toute la complexité de sa tâche, en soulignant dans la préface à sa première traduction de Mallarmé que détruire l'ambiguïté et l'obscurité de ses poèmes veut dire enlever à la poésie mallarméenne ce qu'elle a de caractéristique (1972: 3).

Exprimant ses réserves envers l'idée d'une lecture herméneutique à travers la traduction, Doinaş s'appuie sur sa pratique traduisante et prend position contre une exégèse partisane; il privilégie la «potentialité » des sens dans le processus du traduire et croit à une traduction poétique qui permet «toutes les lectures possibles»:

[...] il me semble que la tâche du traducteur de poésie n'est pas celle de dégager le sens du texte et de le transporter dans le contexte d'une autre langue; une telle ambition herméneutique, explicative ne saurait relever de la conscience de l'acte de traduire, qui est purement applicative, opérationnelle; la tâche du traducteur est de refaire dans sa langue le contexte même de l'original, ce contexte où le sens, où les sens (surtout dans le cas de la poésie) reposent dans leur pure potentialité. Dans cette perspective, la traduction n'est pas une des lectures possibles de l'original, mais - en tant que fidèle re-production du contexte verbal dans l'«épaisseur» de ses structures - précède et garantit toutes les lectures possibles. (Doinaş 1988: 545, souligné dans le texte, traduction de l'auteure) $)^{5}$

Dans ce sens, Doinaş accorde une attention particulière à la «poétique de l'original» et au poiein créateur qui doit être connu et compris mais non pas explicité par le traducteur:

Re-faire la poétique de l'original signifie opérer à ce niveau de la production du texte où les diverses significations se trouvent encore inextricablement unies dans un tronc commun, comme de simples virtualités. Ces dernières vont s'actualiser et se diversifier à la lecture. Cela signifie comprendre sans expliciter, garantir tout comme dans l'original - la possibilité de l'«œuvre ouverte» à une lecture plurielle. (Doinaş 1988: 557, traduction de l'auteure) ${ }^{6}$

En nous ralliant à cette vision de la traduction qui rend possible une lecture plurivoque du texte marqué par l'incertitude, nous réunissons dans la partie analytique qui suit quelques textes poétiques de Mallarmé, Valéry, Tzara et Luca pour étudier la manière dont ils préservent dans la traduction leur ambiguïté d'origine, grâce aux stratégies des traducteurs, adaptées, pour la plupart, aux voix poétiques à rendre, ainsi qu'aux spécificités de la langue et de la littérature d'accueil. 


\section{Traduire l'ambiguïté globale et l'ambiguïté ponctuelle du texte poétique moderne}

Nous commençons par une analyse comparative, centrée sur cette problématique particulière de l'ambiguïté, ayant comme objet le sonnet Salut de Mallarmé. Dans notre article, nous considérons que l'ambiguïté globale ou l'ambiguïté (au singulier) concerne le texte dans son entier, se situe au niveau macrotextuel et lui assure plusieurs lectures, tandis que l'ambiguïté ponctuelle ou les ambiguïtés (au pluriel) se situent au niveau microtextuel, local et concernent l'obscurité et la plurivalence d'une unité de traduction. Ces dernières peuvent, en général, augmenter ou atténuer l'ambiguité et la plurivalence du texte dans son ensemble, les perturber mais non pas les annuler. Nous nous proposons donc d'analyser aussi ce jeu entre ambiguïté globale et ambiguïté ponctuelle, la mesure dans laquelle l'une potentialise ou affaiblit l'autre.

Nous avons choisi trois versions élaborées par trois grands traducteurs roumains - Philippide ${ }^{7}$, Doinaş ${ }^{8}$ et Foarță ${ }^{9}$ - des voix poétiques importantes en Roumanie et qui ont réfléchi et écrit sur la poésie de Mallarmé et sur sa traduction. Leur profil de praticiens et de théoriciens de la traduction qui ont bien compris que l'essentiel est de rendre, selon une expression de Bonnefoy, la "transgression poétique » accomplie par leurs poètes respectifs, profil valable aussi pour les traducteurs des textes de Valéry et de Tzara, font d'eux des figures appropriées pour illustrer une «traductologie de plein champ ». Selon Bonnefoy, la poésie est un "champ de la transgression» des concepts, ce qui la place «sous le signe du spécifiquement poétique» et la rend différente de l'«ordinaire discours» (Bonnefoy 2000: 33-38, 49).

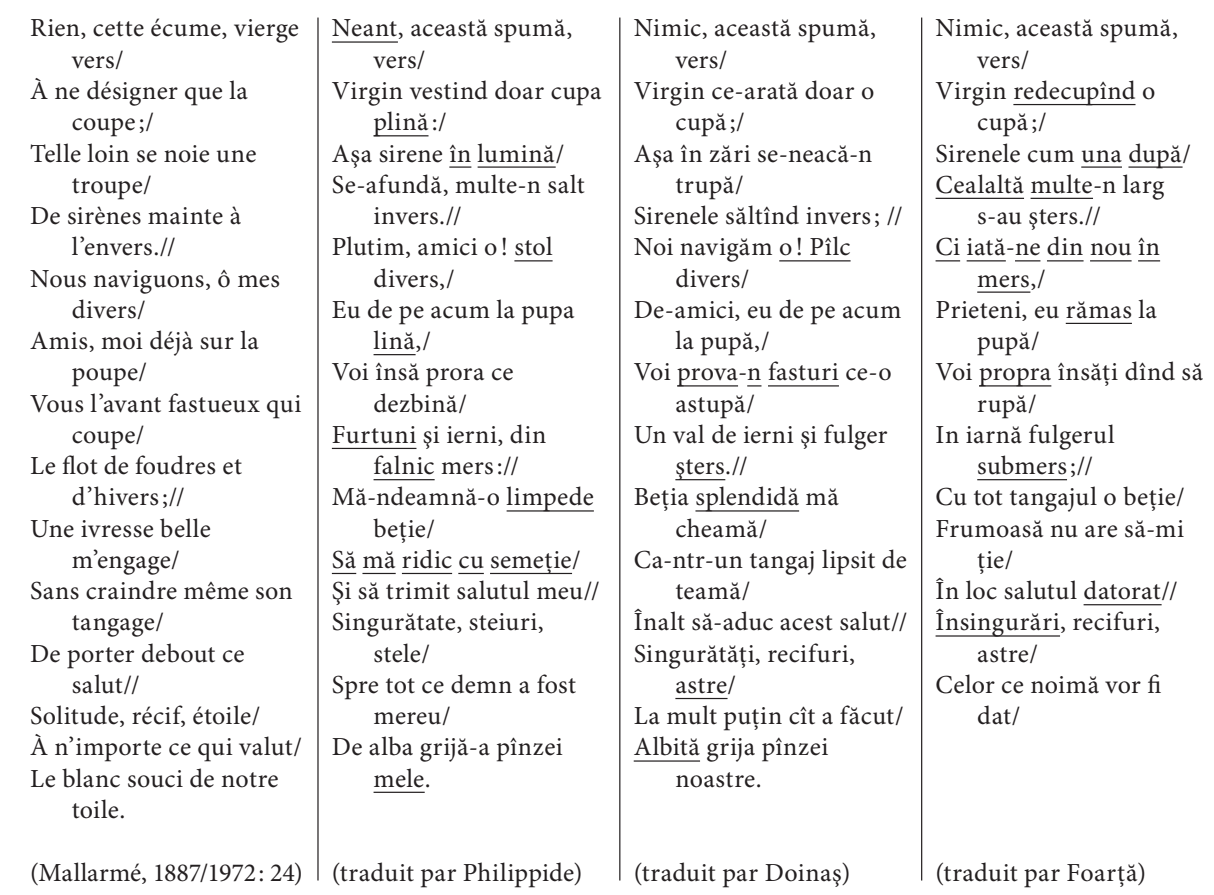




\subsection{Salut de Mallarmé dans la version de Philippide}

Philippide, traducteur également de Baudelaire, Novalis et Rilke, avoue dans ses écrits (1972) que Mallarmé est le plus difficile à traduire de tous les poètes du monde. La difficulté de traduire Mallarmé vient, selon lui, du fait que le poète français a élaboré, avec les mots connus de sa langue, un langage nouveau qui lui est propre et qu'il exprime avec précision «l'imprécis» et «l'énigmatique» (1972: 262-263). Philippide se montre très sensible à l'ambiguïté et aux ambiguïtés qui nous préoccupent ici. Il trouve que chez Mallarmé, on a affaire à «des ambiguïtés captivantes», mais qu'il s'agit en général, dans sa poésie, d'une ambiguïté profonde qui exige des « re-lectures» et qui «engendre des délices intellectuellement poétiques» (1972: 266).

Au bout de quelques décennies de polissage des sept textes de Mallarmé qu’il rend en roumain, Philippide estime que sa «traduction a été travaillée avec la plus grande attention en ce qui concerne le rythme, la rime et surtout l'harmonie intérieure du vers» $(1972: 264)$.

Dans sa chronique sur les versions de Philippide, Doinaş (1974c) fait une critique des traductions nuancée; il pense que la «conscience classique» de Philippide, due à sa formation classique, le pousse, par endroits, à un "relatif sacrifice de l'obscurité génuine» de l'original (1974c: 181), mais il apprécie que, globalement, le poète-traducteur réussisse à rendre «tout le spectre sémantique» de l'écriture mallarméenne (1974c: 181). Doinaș emploie, dans l'article cité, le syntagme «obscurité génuine» avec le sens d'obscurité initiale, authentique, non-dénaturée, obscurité qu’il oppose à un hermétisme recherché.

Si l'on compare l'original et la version de Philippide, on constate que le traducteur a préservé l'ambiguïté et la pluralité des sens de Salut, renvoyant à plusieurs isotopies (banquet, navigation, écriture) (Greimas: 1972), tout en gardant la structure du sonnet, ses contraintes prosodiques, leurs subtilités. Il suit aussi d'assez près la syntaxe elliptique du poète français ayant sa part dans l'obscurité et l'ambiguïté du texte. Quelques ajouts et changements qui retravaillent légèrement le «spectre sémantique» sont, malgré cela, à signaler; rien n'est pas rendu par le mot correspondant en roumain nimic mais par néant, qui introduit des connotations différentes. Loin est rendu, vraisemblablement pour des raisons de rime, par în lumină [dans la lumière], tout comme la coupe reçoit un adjectif plină [pleine], inexistant dans l'original. L'adjectif belle qui caractérise ivresse est remplacé par limpede [claire, limpide], ce qui fait glisser le syntagme vers l'oxymoron et créé une ambiguïté et une obscurité nouvelles dans le texte traduit. Le flot de foudres, unité assez obscure, est rendu par un seul mot, furtuni [tempêtes], solution neutralisante, et tangage est éliminé et remplacé par une

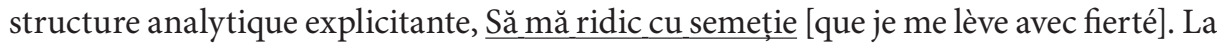
plus surprenante modification, due, probablement, toujours à des raisons d'euphonie, est le remplacement dans le dernier vers du possessif notre auprès du nom toile, par mele [ma], singulier qui restreint, sans doute, sa portée, plus grande dans l'original. C'est aussi le plus important reproche à faire à cette version, qui a le mérite de garder l'ambiguïé de sens globale de l'original, même si telle ambiguïté ponctuelle (le flot de foudres) est atténuée et telle autre (limpide ivresse) est engendrée. 


\subsection{Salut de Mallarmé dans la version de Doinaş}

Doinaş, qui apprécie et admire le travail de Philippide, est lui aussi traducteur de Mallarmé; il en publie des versions roumaines à partir des années 60 et jusqu'en 1988, lorsqu'il inclut Mallarmé dans son Atlas de sons fondamentaux (1988: 219-232). Ces derniers sont pour le traducteur roumain, d'une part, les voix poétiques fortement individualisées qui constituent le patrimoine lyrique de la culture poétique universelle et, d'autre part, un signifiant longuement travaillé selon des contraintes prosodiques. Ce n'est donc pas étonnant que pour ce traducteur de Mallarmé, un but important à atteindre est la restitution en roumain de son «palais sonore impalpable» (Doinaş 1974c: 182), malgré les quelques distorsions de sens que cela suppose.

Il est à remarquer qu'entre la version de 1972 et celle de 1988 de Salut, il y a des différences qui montrent un travail de type mallarméen sur le texte, où toute tentative d'explicitation et de rime facile est corrigée. Malgré quelques différences de pied, dues aux spécificités de la langue roumaine et à une pratique spécifique du sonnet dans la littérature d'accueil, Doinaş réussit le pari de préserver les «savantes, riches et raffinées allitérations, les rimes finales et intermédiaires» (1988:541), tout aussi bien que l'«obscurité génuine» déjà évoquée. Cette dernière nous intéresse particulièrement car nous l'associons à l'incertitude et à l'ambiguïté. D'ailleurs, comme on l'a déjà vu, dans sa préface de 1972, Doinaş parle de la tentation de décodage subie par le traducteur et qui conduit à la destruction de «l'ambiguïté», et, par conséquent, de la poésie mallarméenne (1972: 16).

Doinaş reste plus près de l'original que son prédécesseur pour ce qui est du «spectre sémantique» du texte; on y remarque seuls deux ajouts dont l'un dans o! Pîlc divers/ De-amici [ô! Groupe divers d'amis] pour rendre, ô mes divers/ Amis où pîlc, signifiant groupe, ne nuit pas au sens de l'original et s'explique par des raisons prosodiques. Ces dernières justifient également l'introduction de l'adjectif șters [effacé, éteint, éloigné], postposé à fulger [foudre], solution créative, en fait, qui ajoute des connotations nouvelles, assez ambiguës et obscures dans ce contexte. Ces ambiguïtés nouvelles, situées au niveau local d'une unité, amplifient l'ambiguïté globale de l'original, gardée dans l'ensemble du texte traduit. La belle ivresse devient chez Doinaş splendide, en intensifiant le caractérisant, tandis que l'unité Vous l'avant fastueux qui coupe est rendue en termes maritimes par Voi prova-n fasturi ce-o astupă [Vous la proue en fastes qui comble], où l'avant est traduit par prova [proue]. La seule réserve que nous exprimons envers sa version est la traduction par albită [blanchie] grija pour rendre le blanc souci, épithète qui réduit quelque peu le pouvoir de suggestion du terme original.

\subsection{Salut de Mallarmé dans la version de Foarță}

La dernière version choisie par nous, celle du poète Foarță, comporte plusieurs modifications qui perturbent, au niveau global, la plurivalence des sens contenus dans l'original. Le traducteur s'éloigne de l'original en enlevant ou en ajoutant des connotations et en engendrant des ambiguïtés nouvelles, ponctuelles. C’est le cas du deuxième vers, $A$ ne désigner que la coupe, qui lui inspire une solution créative, légèrement ludique, redecupînd o cupă [redécoupant une coupe], ajoutant à l'ingéniosité phonique une obscurité nouvelle. 
L'unité troupe de sirènes mainte est, au contraire, explicitée et détaillée par le traducteur roumain dans Sirenele cum una după/ Cealaltă multe [comme sirènes l'une après l'autre maintes]; à l'envers est supprimé, ce qui enlève de l'ambiguïté du vers. Dans la deuxième strophe, Nous naviguons, ô mes divers/ Amis, le verbe naviguer est remplacé par Ci iată-ne din nou în mers [nous voilà de nouveau en marche], qui atténue l'isotopie maritime du texte. Le verbe craindre dans l'entourage de tangage est supprimé, affaiblissant de la sorte l'opposition avec la belle ivresse; dans le vers suivant disparaît debout dans De porter debout ce salut, ce qui estompe l'idée de toast, que le traducteur rappelle dans ses gloses comme le premier titre du sonnet (Foarță 1988: 240). Dans le dernier vers, le blanc souci de notre toile, souci est rendu par rîvnă, mot ressenti comme vieilli en roumain (à la différence de souci, en français); son sens renvoie à effort, ardeur, zèle et s'éloigne de l'inquiétude, contenue dans le vocable original souci. Cette version roumaine est un beau poème, finement travaillé, mais un peu trop marqué par la griffe du poète roumain. Le traducteur Foarță entre en compétition avec le poète Foarță, et les deux positions se trouvent dans un équilibre fragile.

Malgré les quelques pertes et ajouts, ces trois versions roumaines nous font pénétrer dans l'univers de Mallarmé, car les trois traducteurs ont su, plus ou moins, garder la plurivalence des sens et une certaine obscurité qui constituent l'incontestable marque mallarméenne. Par endroits, à l'ambiguïté globale de l'original se sont ajoutées des ambiguiités nouvelles, ponctuelles, qui, comme on l'a constaté, intensifient ou atténuent la première, mais sans la perturber gravement.

\subsection{Les Grenades de Valéry dans les versions de Solomon et de Doinaş}

On retrouve la difficulté de rendre la volonté d'expression, une syntaxe spécifique, ainsi que la pluralité des sens - beauté sensuelle d'un fruit, éloge de la perfection, le sens secret des choses, etc. - dans la traduction des Grenades de Paul Valéry ${ }^{10}$ (1929/1978: 97), sous la plume de deux traducteurs de poésie, Petre Solomon ${ }^{11}$ et, de nouveau, Doinaş ${ }^{12}$. Ce dernier connaît bien la «machine de mots» qui préoccupe Valéry, car il réalise, au bout d'un long travail, l'édition critique de son œuvre en roumain (Valéry, 1988), tout en traduisant nombre de ses textes en prose ou en vers.

\begin{abstract}
Dures grenades entr'ouvertes/ Cédant à l'excès de vos grains,/ Je crois voir de fronts souverains/ Eclatés de leurs découvertes!// Si les soleils par vous subis/

Ô grenades entre-baillées,/

Vous ont fait d'orgueils travaillées/

Craquer les cloisons de rubis,//

Et que si l'or sec de l'écorce/

À la demande d'une force/

Crève en gemmes rouges de jus,//

Cette lumineuse rupture/

Fait rêver une âme que j'eus/

De sa secrète architecture.
\end{abstract}

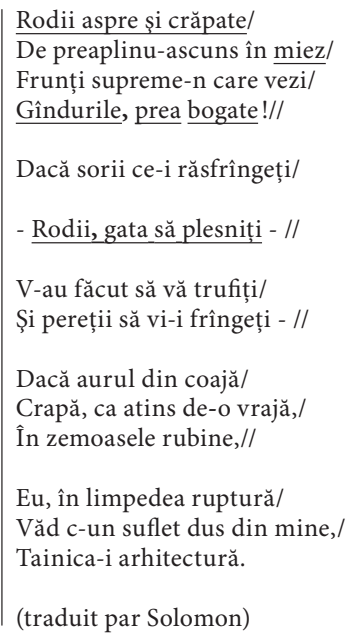

Rodii aspre şi crăpate/ De preaplinu-ascuns în miez/ Frunți supreme-n care vezi/ Gîndurile, prea bogate!//

Dacă sorii ce-i răsfrîngeți/ - Rodii, gata să plesniți - //

V-au făcut să vă trufiți/ Şi pereții să vi-i frîngeți - //

Dacă aurul din coajă/ Crapă, ca atins de-o vrajă,/ În zemoasele rubine,//

Eu, în limpedea ruptură/ Văd c-un suflet dus din mine,/ Tainica-i arhitectură.

(traduit par Solomon)

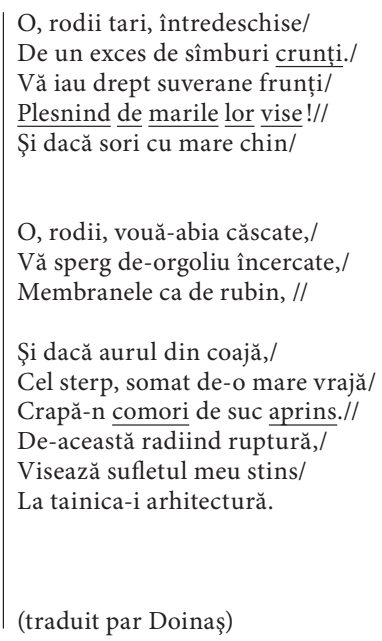


Poète lui-même et traducteur de poésie française, Petre Solomon modifie par endroits le registre de l'original car il rend, par exemple, l'unité Dures grenades entrouvertes par Rodii aspre și crăpate [grenades rugueuses et craquelées], qui donne une image dévalorisante des fruits aimés par Valéry, image renforcée par une autre solution peu appropriée Rodii, gata să plesniți [grenades sur le point de crever] qui est censée rendre Ô grenades entre-baillées. Dans le deuxième vers, l'image des grains est remplacée par une périphrase explicitante, De preaplinu-ascuns în miez [ce qui est caché par la pulpe]. En revanche, dans les tercets, le poète reste près du texte de Valéry et rend bien le son de sa poésie ainsi que l'épaisseur du sens.

Comme on le sait, Valéry pensait que ses vers ont le sens qu'on leur prête, mais nous croyons qu'en traduisant, le traducteur ne doit pas prêter un seul sens au texte traduit, autrement dit, en imposer une seule lecture au public d'accueil, mais lui proposer un texte qui permette différentes lectures à partir d'un texte très proche de l'original. Dans ce sens, la suggestion d'image dévalorisante, induite par le changement de registre dans les deux quatrains, introduit une isotopie étrangère au texte, qui détonne avec l'image de la «secrète architecture», affaiblit la plurivalence des sens et ne conduit pas à ce qu'on a appelé une «interprétation juste»: «Une traduction réussie est celle qui permet une interprétation juste; sa relation avec son original relève de la similarité divergente. Lorsque cette relation relève de la divergence radicale, l'interprétation ne peut être que fausse» (Hewson 2012: 266). Nous pensons que dans ce cas, l'équilibre entre ambiguïté globale et ambiguïté(s) ponctuelle(s) est rompu et le réseau thématique de l'original est affaibli et perturbé par la traduction.

La version de Doinaş reste près de l'original, sans perturbations de registres et de sens, en gardant beaucoup de la perfection formelle et de la plurivalence des sens du sonnet de Valéry. Dans ses notes du traducteur, Doinaş reconnaît avoir préféré le mot vise [rêves], à découvertes dans le quatrième vers pour des raisons de rime (1988: 220). Il avoue certaines modifications également pour d'autres textes de Valéry, ce poète si intéressé par la «substance sonore». Pour le traducteur Doinaş, le crime à commettre contre Valéry est celui de «lèse-musicalité» (1988: 46), ce qui justifie quelques distorsions sémantiques censées ne pas sacrifier la "nécessité musicale intrinsèque» (1988: 48) du texte poétique. Comme les contraintes prosodiques et les différences entre les langues conduisent inévitablement à des ajouts, à des pertes ou à des changements de sens, on signale quelques solutions ponctuelles de ce type qui ont le mérite de ne pas affaiblir l'ambiguïté globale du texte, comme l'ajout de l'épithète crunți postposée au nom sîmburi [grains]; ce choix est créatif car crunt signifie terrible et rarement ensanglanté, d'où une suggestion obscure de couleur rouge, réveillée par le choix audacieux du traducteur. On remarque aussi la métonymie aprins [vif] pour rendre la couleur rouge des gemmes et la traduction de ce dernier mot par un autre plus général comori [trésors], solution neutralisante, mais acceptable. La rareté du terme crunți, avec la connotation couleur $d u$ sang mais aussi dureté, choisi, sans doute "par la magie de la rime», montre qu'une nouvelle ambiguité locale, microtextuelle, peut surgir dans la traduction et peut contribuer à la potentialisation du macrotexte. Comme le traducteur le dit, d'ailleurs, une certaine «obscurité» dans sa traduction se veut un hommage à l'original et au poète, qui rejetait toute explicitation de ses poèmes (Doinaş 1988: 47). La traduction de Doinaş est une preuve que le texte du traducteur peut être tout aussi nuancé et précis que celui de l'auteur, ce qui nous fait penser à Cioran, qui voyait en Valéry un «galérien 
de la Nuance» qui surveille et pèse les mots en une «recherche épuisante de la précision infinitésimale» (Cioran 1992: 90).

\section{Traduire et auto-traduire l'ambiguïté totale des textes dadaïstes et avant-gardistes}

Les quelques esquisses d'analyse comparative des textes de Mallarmé et de Valéry centrées sur le problème de l'ambiguïé globale et ponctuelle montrent que la plupart des traducteurs évoqués essaient de rester très près de l'original au niveau de la syntaxe, de sacrifier le moins possible des mots du texte poétique et d'en préserver la plurivalence des sens. Avec l'avant-garde, pris au sens large du terme, et ses nombreux courants, les choses se compliquent encore, ce qui nous conduit à identifier des catégories très spécifiques de traduction pour ce type de textes.

\subsection{Les textes de Tzara - traduction quasi-littérale, traduction programmée}

Même si la forme du texte poétique se libère souvent des contraintes prosodiques, la pluralité des sens peut aller jusqu'à une ambiguité déconcertante, «absolue», «totale» «infinie», comme le montre la question formulée par Henri Béhar, exégète, par ailleurs, de Tristan Tzara et éditeur des œuvres complètes du poète dadaïste: "Comment réagir devant l'ambiguïté de tout son discours, devant son incohérence syntaxique?» (Béhar 1975: 503, nos italiques).

Irina Mavrodin (2012c: 111-120) donne, indirectement, une réponse de théoricienne et de praticienne de la traduction à cette question, en affirmant que la traduction d'un poème dadaïste, surréaliste ou avant-gardiste se prête bien à un rendu littéral, en fait, quasi-littéral, comme nous le verrons plus loin. Elle prend à l'appui un poème (Retraite) de Tristan Tzara qui aurait beaucoup à perdre par une interprétation ou une rationalisation, pour ne plus parler d'une désambiguïsation, impossible dans un tel cas. Selon la poéticienne roumaine de la traduction, le poème choisi par elle et tiré du volume Vingt-cinq poèmes ${ }^{13}$ nous plonge «dans un étonnement (concept cher à l'esthétique dadaïste et, ultérieurement, à l'esthétique surréaliste) infini, vu la pluralité des sens qui va jusqu'à une ambiguïté totale, permettant une infinité de lectures» (Mavrodin 2012c: 113).

La traductrice roumaine en propose une traduction presque littérale ${ }^{14}$, en expliquant elle-même que la marge de manœuvre du traducteur est limitée au choix des synonymes (arbres pourrait être rendu par arbori, copaci ou pomi) ou à celui d'une forme verbale identique au présent de l'indicatif et à l'impératif, car le contexte n'oriente aucunement ses options. D'où sa conclusion que le type de traduction à choisir est imposé par l'original: «la traduction, est, lorsqu'on procède correctement (ce qui veut dire dans l'esprit de la poétique dadaïste) programmée d'avance (sic), les variations possibles étant très peu nombreuses» (Mavrodin 2012c: 117). Nous comprenons le syntagme utilisé par Mavrodin à propos d'un texte dadaïste, notamment "traduction programmée», comme une traduction qui ne donne pas vraiment au traducteur la possibilité de choisir entre plusieurs solutions sans s'éloigner gravement de la poétique dadaïste, qui suppose justement l'arbitraire des coups de dictionnaire dans le choix des mots poétiques. 


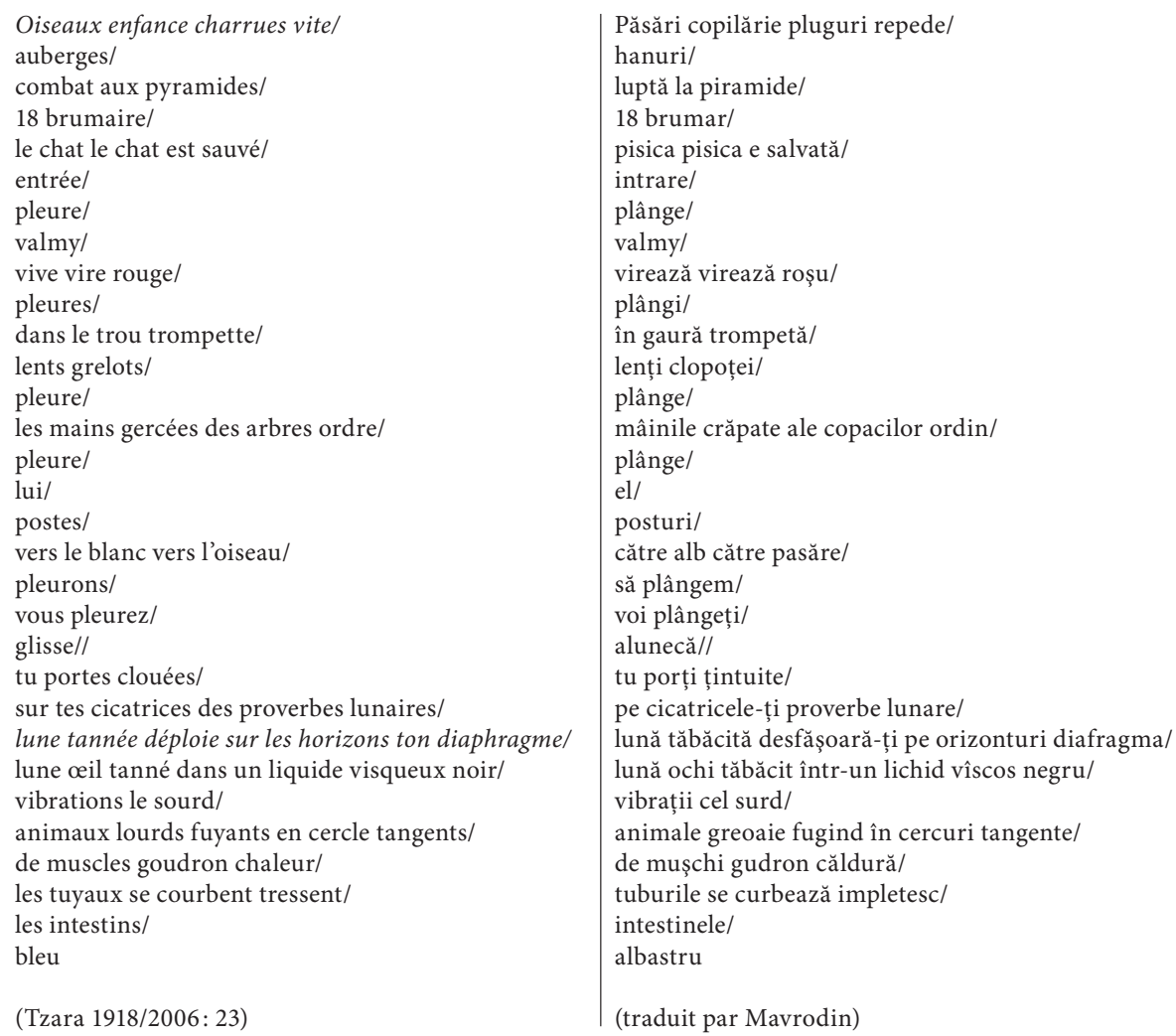

Quelques exemples illustrent bien ce type de contrainte traductive qui permet tout au plus l'oscillation entre un rendu littéral et un rendu presque littéral. Ainsi, le vers inaugural Oiseaux enfance charrues vite est rendu de façon littérale par Păsări copilărie pluguri repede, où seul le mot vite permet des synonymes comme rapidement, mais ce dernier ne contribuerait pas véritablement à faire augmenter/diminuer l'étonnement escompté par le poète de la part de son lecteur. En revanche, un vers comme lune tannée déploie sur les horizons ton diaphragme permet, par la forme verbale déploie qui couvre également l'indicatif présent troisième personne du singulier et l'impératif deuxième personne du singulier, le choix entre ces deux solutions. Mavrodin opte pour l'impératif lună tăbăcită desfăşoară-ți pe orizonturi diafragma, sans trahir pour autant la poétique dadaïste ou l'ambiguïté et la plurivalence du texte. Ces dernières restent entières dans un des vers suivants, de muscles goudron chaleur, qui exige, de nouveau, une solution littérale: de muşchi gudron căldură. Le poème de Tzara, ainsi que sa traduction mavrodinienne, nous laisse voir que «la poésie est le lieu de l'ambiguïté infinie [...] ambiguïté construite par le poète, qui se trouve dans cet état de totale ouverture qu'est l'étonnement, ambiguïté dont peut jouir uniquement un récepteur qui se trouve dans le même état de totale ouverture, d'étonnement.» (Mavrodin 2012c: 116). 


\subsection{Les textes de Gherasim Luca - auto-traduction, réécriture et ambiguïsation}

Même si la poésie dadaïste est un cas extrême, nous pourrons constater que la poésie nommée avant-gardiste sollicite souvent, elle aussi, une traduction littéraliste et même une ambiguïsation plus forte à travers la traduction, comme c'est le cas des textes roumains de Gherasim Luca auto-traduits vers le français.

Comme l'observe l'un de ses exégètes (Toma 2004), les textes Inventatorul iubirii [L'Inventeur de l'amour] et Moartea moartă [La Mort morte] ${ }^{15}$, rédigés en roumain en 1945 et auto-traduits en français en 1994 sous le titre fédérateur L'Inventeur de l'amour suivi de La Mort morte ${ }^{16}$, se tiennent près de l'original; c'est une version littéraliste, "traduction exigée par la nature même du texte: discursivité, transitivité» (Toma 2004: 53-54), où les seules modifications sont la disposition graphique en vers du texte et la suppression des virgules, ce qui fait accroître encore son ambiguïté.

\footnotetext{
Pentru ca iubirea să piardă caracterul paralizant al mamei traumatice şi al complicelui ei compromițător şi castrant care e tatăl, iubita spre care aspiră ființa mea minată, cristlizată și devorată de atâta sete, inepuizabilă de iubire nu poate fi decât o femeie nenăscută
}

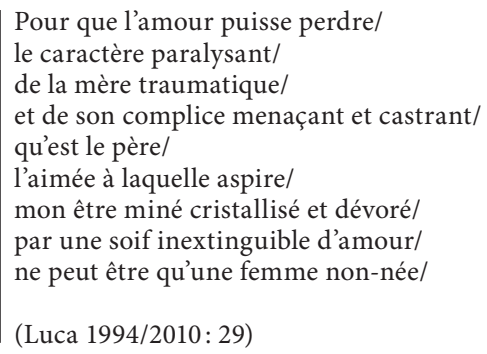

(Luca 1994/2010: 29)

Les choses changent avec Un lup văzut printr-o lupă [Un loup vu à travers une loupe], datant de $1942^{17}$ et traduit en français seulement en 1998, sous le titre Un loup à travers une loupe ${ }^{18}$; ce n'est plus une traduction littéraliste mais une "qui non seulement bouleverse les phrases, mais intervient sur les images et élimine les passages superflus ou explicatifs » et procède à la suppression des comparaisons, à une "ambiguïsation » terminologique et à l'élimination de tout principe explicatif (Toma, 2004: 55-56). Tous ces procédés, identifiés par l'exégète, contribuent à une obscurité voulue et recherchée par le poète qui revient sur ces textes, quatre décennies après leur élaboration en roumain, et procède à une réécriture où la créativité s'épanouit en amplifiant et en intensifiant l'ambiguïté de l'original, ou pour mieux dire, du "premier original».

Dans les exemples suivants, le comparatif ca [comme] est supprimé et la comparaison est remplacée par une métaphore, parfois complétée et amplifiée dans ce qu'on a nommé le «second original» («[...] un texte auto-traduit est-il vraiment à proprement parler une traduction? [...] à supposer qu'il s'agisse encore de traduction, que penser du statut du texte auto-traduit: l'original étant défini non seulement comme la source de l'œuvre mais également comme émanant de l'auteur, une auto-traduction n'est-elle pas un «second original»?, (Oustinoff 2001: 12); ainsi «la femme comme un arbre» devient "cette femme à moitié léopard, bel arbre», "je te porte comme une auge» se transforme en "Je te porte sur mes bras, auge» et "comme un fumeur d'opium» change en «je fume l'opium» : 


\begin{tabular}{|c|c|c|}
\hline $\begin{array}{l}\text { Împing cu palmele întinse ca un } \\
\text { somnambul întunerecul prin } \\
\text { care umblu cu o singură } \\
\text { lanternă această femeie pe care } \\
\text { o simt lângă mine ca pe un } \\
\text { arbore. }\end{array}$ & $\begin{array}{l}\text { [Je pousse avec les paumes } \\
\text { ouvertes comme un } \\
\text { somnambule l'obscurité que } \\
\text { je traverse avec une seule } \\
\text { lanterne cette femme que je } \\
\text { sens près de moi comme un } \\
\text { arbre.] }\end{array}$ & $\begin{array}{l}\text { Somnambule, les paumes ouvertes, } \\
\text { je pousse l'obscurité, ma seule } \\
\text { lanterne: cette femme à moitié } \\
\text { léopard, bel arbre. }\end{array}$ \\
\hline $\begin{array}{l}\text { [...] te port pe brațe ca pe o albie } \\
\text { în care se scaldă un şarpe. }\end{array}$ & $\begin{array}{l}\text { [...] [je te porte sur mes bras } \\
\text { comme une auge où se } \\
\text { baigne un serpent.] }\end{array}$ & $\begin{array}{l}{[\ldots] \text { je te porte sur mes bras, auge }} \\
\text { où on lave les serpents. }\end{array}$ \\
\hline $\begin{array}{l}{[\ldots] \text { singur în camera mea ca un }} \\
\text { fumător de opium respirând } \\
\text { carnea ta } \\
\text { îndepărtată }[\ldots]\end{array}$ & $\begin{array}{l}{[\ldots] \text { [seul dans ma chambre }} \\
\text { comme un fumeur d'opium } \\
\text { respirant ta chair.] }\end{array}$ & $\begin{array}{l}{[\ldots] \text { seul dans ma chambre, je fume }} \\
\text { l'opium de ta chair lointaine. }\end{array}$ \\
\hline $\begin{array}{l}\text { Simbolul } \\
\text { frumos ca un vampir, îmi face } \\
\text { semne secrete } \\
\text { cu scheletul ei. }\end{array}$ & $\begin{array}{l}\text { [Le symbole } \\
\text { beau comme un vampire me fait } \\
\text { des signes secrets } \\
\text { avec son squelette.] }\end{array}$ & $\begin{array}{l}\text { O doux symbole, d'un gant ou bien } \\
\text { de son souple squelette, vous me } \\
\text { faites signe et le collier de dents } \\
\text { d'un vampire brille au cou du } \\
\text { réel. }\end{array}$ \\
\hline $\begin{array}{l}\text { (Luca 1945/2003: 171, 173, 178, } \\
\quad 174)\end{array}$ & (Luca [traduction de l'auteure]) & (Luca 1998-2010: 8, 10, 15, 10) \\
\hline
\end{tabular}

Dans cette dernière séquence l'auto-traducteur procède à un étoffement qui rend l'image plus complexe et obscure tandis que les ajouts font accroître le bizarre, car «beau comme un vampire» devient "le collier de dents d'un vampire brille au cou du réel», solution que, du point de vue de la liberté et de l'étrangeté, seul un autotraducteur avant-gardiste peut se permettre.

On remarque, ailleurs, le phénomène d'une véritable réécriture plus ambiguë, où le texte se dilate et s'intensifie par rapport au texte roumain, fait accompagné d'un changement surprenant d'images. On y trouve même une traduction qu'on pourrait nommer "paradoxale», d'un élément par son contraire, comme dans ce passage du Château pressenti du même volume, où «întunericul» (le noir, l'obscurité) est rendu par la lumière, ce qui justifie pleinement le titre de «second original» donné à l'autotraduction.

Întunericul cu care se înconjoară această apariție face ca noaptea să fie întotdeauna o noapte fără lună, o noapte pe care am început să o recunosc mai mult cu vîrful degetelor, $\mathrm{cu}$ pleoapele, pot să pipăi noaptea, o pot săruta ca pe o femeie dar apariția asta mai inconsistentă decît punctul, decît fumul, îmi alunecă printre degete, efortul de a apuca, de a prinde, surprinzîndu-mă în mijlocul camerei cu o mînă strînsă puternic în jurul gîtului, oniric început de agonie!
[L'obscurité dont s'entoure cette apparition fait que la nuit soit toujours une nuit sans lune, une nuit que je commence à reconnaître surtout avec les bouts des doigts, avec les paupières, je peux toucher la nuit, je peux l'embrasser comme une femme mais cette apparition plus inconsistante que la fumée glisse entre mes doigts, l'effort de saisir, de prendre, me surprend au milieu de ma chambre, une main bien serrée autour du cou, onirique début d'agonie!]

(Luca [ (traduction de l'auteure])
La lumière que cet oiseau absorbe prive la nuit de sa principale source de clarté, la lune et les étoiles, et, comme dans le noir qu'il secrète l'obscurité même finit par se dissoudre, c'est en aveugle que je cherche la réalité de la nuit, c'est avec les bouts des doigts et des paupières que je la touche et que, tout haletant, je la serre finalement dans mes bras.

Mais plus friable que le point dans son acception purement théorique, l'oiseau dont je parle, sans pour autant savoir le nommer, rebondit comme une balle, tantôt balle de jeux, tantôt balle de meurtre, et ses coups de feu mêlés à d'énormes éclats de rire affolent encore plus mon besoin de le saisir pour me retrouver peu après hagard au milieu de la nuit et de la pièce, une main autour du cou et plus certain que jamais de l'avoir enfin rattrapé au moment précis où je m'étrangle - onirique début d’agonie!

(Luca 1998/2010: 26-27) 
Dans son «second original», Gherasim Luca enlève la comparaison «je peux embrasser la nuit comme on embrasse une femme», en rendant l'idée d'embrasser d'une façon plus inattendue avec: "je la serre [la nuit] finalement dans mes bras». Il ajoute une image nouvelle en jouant sur le sens du mot balle, de jeu ou d'arme, incertitude impossible en roumain puisqu'il y a deux termes différents [respectivement minge et glonte] pour les deux référents. Il obscurcit encore le texte par l'introduction de l'image d'un oiseau "plus friable que le point dans son acception purement théorique» et par le mélange de "coups de feu mêlés, à d'énormes éclats de rire».

$\mathrm{Au}$ bout de ces esquisses d'analyse sur les textes de Tzara et de Luca, on peut dire que les textes d'avant-garde, qui jouent sur une ambiguïté infinie, sollicitent des stratégies de traduction spécifiques - traduction programmée, traduction paradoxale, réécriture - où le littéralisme, l'ambiguïsation et l'obscurcissement ont une place de choix.

\section{Conclusion}

Les exemples assez variés et nombreux, que nous avons pris pour objet d'analyse, montrent que le texte poétique moderne se nourrit souvent de l'ambiguïté que le traducteur se doit de préserver à travers sa traduction, tandis que l'auto-traduction permet même l'ambiguïsation accrue de la version traduite. Des essayistes et des traductologues partagent l'idée que la traduction doit garder entière l'incertitude de choix entre telle ou telle isotopie pour que le texte traduit offre, tout comme l'original, un potentiel de lecture plurielle.

En paraphrasant le grand poète traducteur Bonnefoy, nous pouvons dire que les traducteurs dont nous avons analysé les versions - Philippide, Doinaş, Foarță, Solomon, Mavrodin - ont bien réfléchi à la «transgression poétique» de leurs poètes et ont su la «transposer dans une autre langue», ayant le «courage» et l'«audace» de «ne trahir que l'inessentiel» (Bonnefoy 2000: 49).

Comme on a pu le voir dans les versions roumaines de Mallarmé et de Valéry, le traducteur essaie de rester très près de l'original pour sa plurivalence des sens mais aussi pour sa perfection formelle, tout en gardant des mots poétiques spécifiques à un univers poétique, une syntaxe elliptique modulée à sa manière par le poète, un rythme propre à sa voix poétique. Les risques d'éloignement du texte source sont difficiles à contourner et conduisent souvent à des pertes de connotations, à un ajout de significations, à des neutralisations de vocables ou à l'affaiblissement d'un réseau thématique et, plus rarement, à une atténuation de l'ambiguïté globale, défavorable à l'épaisseur de sens de l'original.

Si, pour des poètes comme Mallarmé et Valéry, on peut parler de l'«ambiguïté globale» et de l'«l'ambiguïté ponctuelle» du texte et du jeu entre macro et microtexte qu'elles entretiennent, pour les poètes d'avant-garde, il semble nécessaire d'y ajouter un superlatif et de parler, pour le cas de Tristan Tzara, par exemple, d'une "ambiguïté totale» «absolue», «infinie» qui sollicite une traduction littérale, étant par cela une «traduction programmée d'avance». Comme les synonymes et les formes verbales identiques donnent une certaine incertitude sur le meilleur choix à faire et comme le contexte n'aide pas le traducteur dans ses hésitations, on peut parler, en fin de compte, d'une traduction "quasi-littérale» d'un poème dadaïste.

Lorsque les poètes avant-gardistes prennent la plume pour s'auto-traduire, comme le fait Luca, l'ambiguité et l'obscurité peuvent être privilégiées et on peut 
même parler d'une «traduction paradoxale», où un mot est rendu par son contraire. Comme l'auto-traduction conduit souvent à une réécriture, autrement dit à un «second original», on peut avoir affaire à une traduction où le texte source est seulement un point de départ qui permet la suppression des passages explicatifs, l'omission des comparatifs autorisant une association de termes, ainsi que l'introduction d'images étonnantes et surprenantes, ou même de nouveaux paragraphes.

Devant de tels textes, le traducteur connaît le doute et l'incertitude, hésitant quant à la solution à choisir pour les rendre dans sa langue afin d'en préserver l'ambiguité, l'obscurité et la plurivalence. Si, pour le traducteur de Mallarmé ou de Valéry, la difficulté est de garder, à la fois, l'«ambiguïté globale» et l'«ambiguïté ponctuelle» et d'équilibrer leur jeu dans le texte traduit, pour le traducteur ou l'autotraducteur de Tzara ou de Luca, le défi est de trouver une solution d'«ambiguïté totale» et/ou d'aller vers la créativité de la réécriture et donner à l'ambiguité une plus grande ampleur et une nouvelle intensité.

\section{NOTES}

* Contribution réalisée dans le cadre du programme CNCS PN-II-ID-PCE-2011-3-0812 (Projet de recherche exploratoire) Traduction culturelle et littérature(s) francophones: histoire, réception, critique des traductions, Contrat 133/27.10.2011.

1. Mavrodin, Irina (2013): Lecture plurielle, ambigüité [Demersul hermeneutic este cea mai greșită strategie, cred eu, când suntem confruntați cu un text de maximă poeticitate, altfel spus de maximă ambigutate] (traduit par Raluca Nicoleta BALAȚCHI). Atelier de traduction, Fragmentarium, 19: 101-103; texte original dans MAVRodin, Irina (2006a): Despre traducere: literal şi în toate sensurile [Sur la traduction: littéralement et dans tous les sens]. Craiova: Scrisul românesc, 21. Nota bene: Irina Mavrodin a publié des textes en roumain mais également en français. Certains de ses textes ont été traduits en français dans la revue Atelier de traduction, dans la rubrique Fragmentarium. Là où il n'y a pas de mention pour le texte original en roumain, le texte a été rédigé et publié en français.

2. Voir leur article dans ce numéro.

3. Mavrodin, Irina (2012b): Une pratico-théorie de la traduction littéraire en dix fragments (traduit du roumain par Cristina DrAhtA). Atelier de traduction, 18: 133-137; texte original: «Eu propun o teorie a traducerii ca lectură plurală, ce rămâne - paradoxal - validă chiar şi atunci când, în cazul unor texte care o cer, traducătorul încearcă să mențină ambiguitatea - deci pluralitatea de sensuri - din original. A opta pentru menținerea ambiguității este, în sine opțiunea pentru o anume lectură. [...] Cititorul (criticul, de asemenea, în calitatea lui de de cititor specializat) re-creează opera prin lectura sa. Acest punct de vedere poate fi extins la traducerea literară şi la activitatea traducătorului literar, în care putem vedea un citittor al cărui efort, materializat în transpunerea operei dintr-o limba în alta, este cel mai aproape de ideea pe care ne-o facem despre lectură ca act creator.» dans Mavrodin, Irina (2006): Despre traducere: literal şi în toate sensurile. Craiova: Scrisul românesc, 93-94.

4. Mavrodin, Irina (2013): Lecture plurielle, ambiguïté (traduit du roumain par Raluca Nicoleta BALAȚCHI). Atelier de traduction, 19: 101-103; texte original: «Dificultatea de a menține ambiguitatea (alt concept important al poeticii moderne) şi totodată de a traduce [...] devine evidentă când avem de-a face cu autori de maximă ambiguitate, cum ar fi Rimbaud, Mallarmé, Char, Michaux, etc. Traducătorul se vede pus în situația de a inventa, crea un text izomorf (alt concept al poeticii) textului original, un text atent la alcătuirea lui, la felul cum este alcătuit, care să permită o lectură plurivocă, şi nu la sensul univoc pe care mereu este tentat sa-l înțeleagă, să-l scoată la suprafață printr-un demers hermeneutic.» dans Mavrodin, Irina (2006a): Despre traducere: literal şi în toate sensurile. Craiova: Scrisul românesc, 21.

5. Texte original: «[...] mi se pare că sarcina traducătorului de poezie nu este aceea de a degaja sensul textului şi de a-l transporta în contextul altei limbi; o asemenea ambiție hermeneutică, explicativă nu pate să țină de conştiința actului de a traduce, care e pur aplicativă, operațională : sarcina traducătorului e tocmai aceea de a reface, în limba sa, tocmai contextul originalului, acela în care sensul, sau sensurile (mai ales în cazul poeziei), se odihnesc în pura lor potențialitate. În 
această lumină, traducerea nu este $\underline{u n a}$ din lecturile posibile ale originalului, ci ca fidelă reproducere a contextului verbal în "grosimea» structurilor sale - le precede şi le garantează pe toate. " (souligné dans le texte)

6. Texte original: «A reface poetica originalului înseamnă a opera la acel nivel al producției de text unde diversele semnificații se află încă unite inextricabil într-un trunchi comun, ca simple virtualități; acestea urmează să se actualizeze şi să se diversifice la lectură. Aceasta înseamnă a înțelege fără a explica, a garanta şi în traducere, la fel ca în original - posibilitatea „operei deschise”, a unor lecturi plurale.» (souligné dans le texte)

7. Mallarmé, Stéphane (1887/1974): Salut (Traduit par Alexandru Philippide). In: Caraion, Ion, Crohmalniceanu Ovid S., Antologia poeziei franceze de la Rimbaud pînă azi. Bucarest: Biblioteca pentru toți, Minerva, vol. I, 36-37.

8. Mallarmé, Stéphane (1887/1988): Salut, (Traduction, préface et postface par Ştefan Augustin Doinaş), Atlas de sunete fundamentale. Cluj-Napoca: Dacia, 221.

9. Mallarmé, Stéphane (1887/1988): Album de versuri. (Traduction et gloses par Şerban FoARŢ̌a). Bucarest: Univers, 26-27.

10. Valery, Paul (1929/1978): Poésies. Paris: Gallimard, 97.

11. Solomon, Petre (1974): «Rodii» in Caraion, Ion, Crohmalniceanu Ovid S. (dirs) (1974, 1975, 1976): Antologia poeziei franceze de la Rimbaud pină azi [Anthologie de la poésie française de Rimbaud jusqu'à présent], Collection Biblioteca pentru toți. Bucarest: Minerva, vol. I, 293.

12. Valery, Paul (1988): Poezii. Dialoguri. Poetică şi estetică [Poésies. Dialogues. Poétique et esthétique]. Édition et préface par DoInAş, Ştefan Augustin. Bucarest: Univers: 140.

13. Tzara, Tristan (1918/2006): Vingt-cinq poèmes. Éditions Dilecta.

14. Tzara, in Mavrodin Irina (2012c): Un texte où littéralement coïncide avec littérairement, Echiquier - essais de poïétique/poétique. Iaşi: Editura Timpul, 111-120.

15. Inventatorul iubirii; Moartea moartă. In: LuCA, Gherasim (1945/2003): Inventatorul iubirii şi alte scrieri, In: Pop Ion. Cluj-Napoca: Dacia, 229-246; 261-284.

16. LucA, Gherasim (1994/2010): L'Inventeur de l'amour suivi de La Mort morte. Paris: José Corti.

17. Un lup văzut printr-o lupă. In: LuCA, Gherasim (1945/2003): Inventatorul iubirii şi alte scrieri, In: Pop Ion. Cluj-Napoca: Dacia, 169-228.

18. LuCA, Gherasim (1988/2010): Un loup à travers une loupe. Paris: José Corti. 1 1998/2010.

\section{RÉFÉRENCES}

Abou Fadel, Gina et Awaiss, Henri, dir. (2005): Pour dissiper le flou, Traduction - Traductologie. Collection Sources-Cibles. Beyrouth: École de Traducteurs et d'Interprètes de Beyrouth, Université Saint-Joseph.

Ballard, Michel (2005): Il n'est pas certain. In: Henri Awaiss et Gina Abou Fadel, dir. Pour dissiper le flou, Traduction - Traductologie, Collection Sources-Cibles. Beyrouth: École de Traducteurs et d'Interprètes de Beyrouth, Université Saint-Joseph, 21-24.

BAlliu, Christian (2005): La traductologie: Une lutte d'influences. In: Henri Awaiss et Gina Aвou FAdel, dir. Pour dissiper le flou, Traduction - Traductologie. Collection SourcesCibles. Beyrouth: École de Traducteurs et d'Interprètes de Beyrouth, Université SaintJoseph, 25-28.

Balliu, Christian (2010): Le traducteur, le médecin et le patient. Meta. 55(1):15-22.

Behar, Henri (1975): Préface. Tristan Tzara, Euvres complètes. Paris: Flammarion.

Benjamin, Walter (1955/2000) : Sarcina traducătorului, Iluminări. (Traduit par Catrinel PLEşU) [La tâche du traducteur, Illuminations]. Bucarest: Univers, 45-55.

Bonnefoy, Yves (2000): La communauté des traducteurs. Strasbourg: Presses Universitaires de Strasbourg.

Caraion, Ion, Crohmalniceanu Ovid S. (dirs) $(1974,1975,1976)$ : Antologia poeziei franceze de la Rimbaud pînă azi [Anthologie de la poésie française de Rimbaud jusqu'à présent], Collection Biblioteca pentru toți. Bucarest: Minerva, vol. I, II, III.

Cioran, Emil (1992): Valéry face à ses idoles. In: Exercices d'admiration - essais et portraits. Arcades. Paris: Gallimard, 73-96.

Clas, André (2005): Traduire c'est tailler un diamant en multiples facettes! In: Henri AwAIss et Gina Aвou Fadel, dir. Pour dissiper le flou, Traduction - Traductologie. Collection 
Sources-Cibles. Beyrouth: École de Traducteurs et d'Interprètes de Beyrouth, Université Saint-Joseph, 49-53.

Delisle, Jean (2005): Les théories de la traduction: une complexité à apprivoiser. In: Henri Awaiss et Gina Abou Fadel, dir. Pour dissiper le flou, Traduction - Traductologie. Collection Sources-Cibles. Beyrouth: École de Traducteurs et d'Interprètes de Beyrouth, Université Saint-Joseph, 69-74.

DoInaş, Ştefan Augustin (1972): Prefață [Préface]. In: Mallarmé, Stéphane (1972): Poezii [Poésies]. (Traduit par Ştefan Augustin DoInAş). Préface de Ştefan Augustin Doinaş. Bucarest: Univers, 3-21.

DoINAş, Ştefan Augustin (1974b): Traducerea ca re-creare a operei [La traduction en tant que re-création de l'oeuvre]. In: Orfeu şi tentația realului. Bucarest: Editura Eminescu, 258-263.

DoINAş, Ştefan Augustin (1974c): Cronica traducerilor, Mallarmé. In: Secolul. 20(5-6):160-161, 181-182.

DoINAş, Ştefan Augustin (1988): Despre traducerea fidelă a poeziei [Sur la traduction fidèle de la poésie]. In: DoINAş, Ştefan Augustin, Atlas de sunete fundamentale. (Traduit par Ştefan Augustin DoInAş), Préface et postface par Ştefan Augustin DornAş. Cluj-Napoca: Dacia.

Eco, Umberto (2003/2008): A spune cam acelaşi lucru, experiențe de traducere [Dire presque la même chose, expériences de traduction]. (Traduit par Alexandru Laszlo). Iaşi: Polirom.

FoArȚă, Şerban (1988): Glose [Gloses].Note[Notes] In: MAllarme, Stéphane, Album de versuri [Album de vers]. (Traduit par Şerban FoArŢ厶̆). Bucarest: Univers, 184-233, 235-262).

Froeliger, Nicolas (2008): Le problème de la nuance en traduction pragmatique. Traduire, 318:77-93.

Hewson, Lance (2012): Équivalence, leurre, divergence. In: Camille Fort et Florence LautelRibstein, dir. Des mots aux actes, 3. Jean-René Ladmiral, une cuvre en mouvement. PerrosGuirec: Anagrammes, 257-270.

Greimas, Algirdas Julien (dir.) (1972): Essais de sémiotique poétique. Paris: Larousse.

Ladmiral, Jean-René (2005): Traduire c'est désambiguïser. In: Henri Awaiss et Gina Aвоu FADEL, dir. Pour dissiper le flou, Traduction - Traductologie. Collection Sources-Cibles. Beyrouth: École de Traducteurs et d'Interprètes de Beyrouth, Université Saint-Joseph, 127-130.

Le Blanc, Charles (2005): L’une des stratégies. In: Henri Awaiss et Gina Abou Fadel, dir. Pour dissiper le flou, Traduction - Traductologie. Collection Sources-Cibles. Beyrouth: École de Traducteurs et d'Interprètes de Beyrouth, Université Saint-Joseph, 131-133.

Mavrodin, Irina (2006a): Despre traducere. Literal şi în toate sensurile. Craiova: Scrisul românesc.

Mavrodin, Irina (2006b): Le faire du traducteur de littérature ou pour une pratico-théorie auctoriale. In: Despre traducere: literal şi în toate sensurile. Craiova: Scrisul românesc, 170-172.

Mavrodin, Irina (2012a): Échiquier - essais de poïétique/poétique. Iaşi: Editura Timpul.

Mavrodin, Irina (2012c): Un texte où littéralement coïncide avec littérairement. Échiquier essais de poḯtique/poétique. Iaşi: Editura Timpul, 111-120.

Mavrodin, Irina (2013): Lecture plurielle (traduit par Raluca-Nicoleta Balațchi). Atelier de traduction. Suceava: Editura Universităţii Suceava, 19:101-103.

Meschonnic, Henri (2005) : Le flou et le clair. In: Henri Awaiss et Gina Abou Fadel, dir. Pour dissiper le flou, Traduction - Traductologie. Collection Sources-Cibles. Beyrouth: École de Traducteurs et d'Interprètes de Beyrouth, Université Saint-Joseph, 147-148.

Oustinoff, Michäel (2013): Bilinguisme d'écriture et auto-traduction - Julien Green, Samuel Beckett, Vladimir Nabokov. Paris: L'Harmattan.

PAnă, Saşa (1969): Antologia literaturii române de avangardă [Anthologie de la littérature roumaine d'avant-garde]. Bucarest: Editura pentru literatură.

Philippide, Alexandru (1972): Considerații confortabile [Considérations confortables], vol. II, Bucarest: Editura Eminescu.

ToмA, Iulian (2004): L’auto-traduction chez Gherasim Luca. In: Atelier de traduction, 1:51-58. 